\title{
Emergency Department Presentations by Children in Remote Australia: A Population-based Study
}

Global Pediatric Health

Volume 8: I-II

(c) The Author(s) 2021

Article reuse guidelines:

sagepub.com/journals-permissions

DOI: 10.1177/2333794X21991006

journals.sagepub.com/home/gph

(S)AGE

\author{
Philippa Jane Dossetor, BMedSc(Hon), MChD ${ }^{1,2} \mathbb{D}$, \\ Emily F. M. Fitzpatrick, MBBS, PhD ${ }^{2,3}$, Kathryn Glass, PhD', \\ Kirsty Douglas, MBBS, MD", Rochelle Watkins, $\mathrm{PhD}^{5}$, \\ June Oscar ${ }^{6,7}$, Maureen Carter ${ }^{8}$, David Harley, MBBS, PhD ${ }^{1,9}$, \\ Heather E. Jeffery, MBBS, PhD $^{2}$, Elizabeth Jane Elliott, MD, MBBS ${ }^{2,3}$, \\ and Alexandra L. C. Martiniuk, BPsych, PhD $2,10,11$
}

\begin{abstract}
Background. Aboriginal leaders invited us to examine the frequency and reasons for emergency department (ED) presentations by children in remote Western Australia, where Prenatal Alcohol Exposure (PAE) is common. Methods. ED presentations (2007-I I inclusive) were examined for all children born in the Fitzroy Valley in 200203. Results. ED data for $127 / 134$ (94.7\%) children (95\% Aboriginal) showed 1058 presentations over 5-years. Most (8I\%) had at least I presentation (median 9.0, range I-50). Common presentations included: screening/follow-up/ social reasons (16.0\%), injury (I5.I\%), diseases of the ear (I4.9\%), skin (I3.8\%), respiratory tract (I3.4\%), and infectious and parasitic diseases (9.8\%). PAE and higher presentations rates were associated. Commonly associated socio-economic factors were household over-crowding, financial and food insecurity. Conclusion. Children in very remote Fitzroy Crossing communities have high rates of preventable ED presentations, especially those with PAE. Support for culturally appropriate preventative programs and improved access to primary health services need to be provided in remote Australia.
\end{abstract}

\section{Keywords}

Aboriginal, emergency department presentation, pediatric, rural and remote, Australia

Received July 25, 2020. Received revised December 17, 2020. Accepted for publication January 8, 2021.

What we already know?

Aboriginal health in Australia has received much research attention, however little focus has been given to community level studies which provide opportunities to influence local policy development and implementation. There are few peer-reviewed publications describing Emergency Department (ED) presentations by Aboriginal children in very remote Western Australia. Furthermore, whole Indigenous Australian pediatric population group studies or studies that describe service use are rare.

What this article adds?

This project aimed to fill these gaps to inform solutions for this and similar communities nationally, and to allow for comparison with similar population
'Australian National University, Canberra, ACT, Australia

'University of Sydney, Sydney, NSW, Australia

${ }^{3}$ The Sydney Children's Hospital Network, Westmead, NSW, Australia

${ }^{4}$ Australian National University, Woden, ACT, Australia

${ }^{5}$ The University of Western Australia, Perth, WA, Australia 'Marninwarntikura Women's Resource Centre, Fitzroy Crossing,

WA, Australia

${ }^{7}$ University of Notre Dame, Broome, WA, Australia

${ }^{8}$ Nindilingarri Cultural Health Services, Fitzroy Crossing, WA, Australia

${ }^{9}$ The University of Queensland, Brisbane, QLD, Australia

${ }^{10}$ University of Toronto, Toronto, ON, Canada

I'The George Institute for Global Health, Sydney, NSW, Australia

Corresponding Author:

Philippa Jane Dossetor, Australian Nation University Medical School Building 4, The Canberra Hospital, Hospital Rd, Garren, Canberra, ACT 2605, Australia.

Email: philippa.dossetor@health.nsw.gov.au 
groups internationally. We aimed to describe the reasons for presentation and outcomes of emergency department presentations to Fitzroy Crossing Hospital by children in the Lililwan cohort $(95 \%$ of all children born between 2002-3 and living in the valley in 2010-2011). As this cohort were comprehensively assessed for Fetal Alcohol Spectrum Disorders (FASD), we hypothesized that a correlation between Prenatal Alcohol Exposure (PAE) or FASD and frequency of emergency department presentations would exist. We found that presentations to emergency department with preventable conditions are frequent for young Aboriginal children in very remote Australian communities, with higher rates in children with PAE however, there was no correlation between FASD diagnoses and higher rates of presentations. This indicates need for culturally appropriate health literacy, better living conditions, and improved access to primary health services and prevention programs.

\section{Background}

Indigenous Australians comprise 3\% of Australia's population and $80 \%$ reside in rural or remote Australia. ${ }^{1}$ In very remote locations, the proportion of the population who are Aboriginal is over $45 \%{ }^{2}$ and in the Fitzroy Valley the proportion is $93 \% .^{3}$ The Aboriginal age pyramid in remote settings is skewed toward children. In 2011, 36\% of Aboriginal Australians were aged 0 to 14 years. $^{2}$

Despite a $33 \%$ decrease in the gap in mortality rates between Aboriginal and non-Indigenous children aged under-5-years from 1998 to 2015, rates remain 3.5 times higher for Indigenous children in Western Australia (WA), at 189 versus 54 per $100,000{ }^{2}$

In the very remote Kimberley region of northern WA lies the Fitzroy Valley, a $2500 \mathrm{~km}^{2}$ area, home to approximately 4500 people. Fitzroy Crossing is the service town for the Fitzroy Valley and the site of the only hospital, servicing about 45 very remote communities within approximately a $200 \mathrm{~km}$ radius. In 2013, 1400 people living in the Fitzroy Valley were children aged under 16 years. ${ }^{3}$ These Aboriginal people belong to 5 main language groups (Bunuba, Walmajarri, Wangkatjungka, Gooniyandi, Kija, and Nyikina) and maintain traditional practices. ${ }^{3}$

In 2016/17 there were 67,032 ED presentation across all Kimberley hospitals. ${ }^{4}$ Fitzroy Crossing Hospital is a 12-bed inpatient facility with an associated emergency department (ED). The hospital is funded for 4 doctors and 15 nurses, however recruitment and retention of staff is challenging in remote Australia, so the workforce did not always have full capacity. On average the hospital was staffed by 3 doctors and 8 nurses. ${ }^{5}$ Five separate consultation rooms are used for outpatient GP and visiting specialist consultations. GP consultations are available within restricted hours, for walk-in visits and a limited number of booked appointments. Some remote communities outside Fitzroy Crossing town have nurserun health clinics and receive intermittent GP services from the RFDS. ${ }^{5}$ The Lililwan Project was a collaborative, community-led, population-based prevalence study of Fetal Alcohol Spectrum Disorder (FASD), Prenatal Alcohol Exposure (PAE), early life trauma and health of children in the Fitzroy Valley that arose from the locally developed Marulu Strategy. 6,7

In Stage 4 of the Lililwan Project we aim to describe reasons for and outcomes of ED presentations to Fitzroy Crossing Hospital by children in the Lililwan (all the little ones in Kimberley Kriol and representing the cohort who underwent complex multi-disciplinary assessments for Fetal Alcohol Spectrum Disorders) cohort, assess for correlations between PAE and/or FASD and frequency of emergency department presentations. We hope to highlight the implications for child health, health care and service delivery in remote Australia, and inform solutions for similar communities by comparison.

\section{Methods}

\section{The Lililwan Project}

This population-based study of children (Lililwan cohort) in the Fitzroy Valley was conducted between 2010 and 2013 (Figure 1). As previously described, the aim of the Lililwan project was to determine FASD prevalence using validated, multi-disciplinary neurodevelopmental assessments. This age group was selected because they were old enough to participate in the FASD assessment, yet young enough to benefit from medical, educational, and behavioral interventions. Also, this cohort was born before the introduction of alcohol restrictions throughout the Fitzroy Valley in 2008 hence PAE was more likely. ${ }^{8}$ Identification and characterization of the cohort, definition of terms, culturally safe consent process utilizing "community navigators" and local languages, and results from the FASD prevalence study (Stages 1\&2) are published elsewhere. ${ }^{8-11}$ Demographic data (Table 2) were gathered during interviews with parents or carers using a standardized data form during Stage $1^{8}$ and FASD diagnoses were made during Stage 2. ${ }^{6}$ In Stage 3 we mapped health services. ${ }^{5}$ In Stage 4 we documented lifetime hospitalizations 


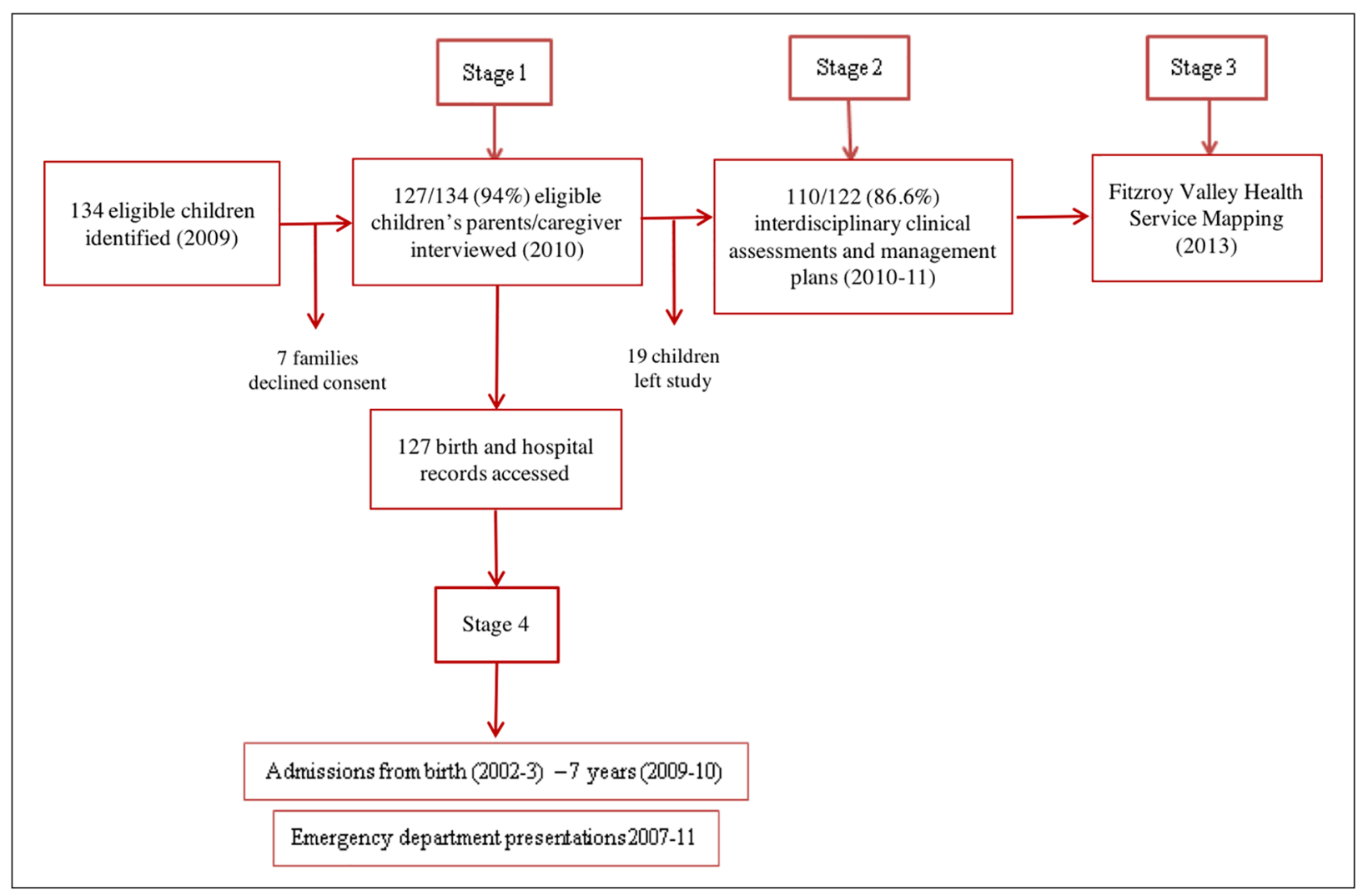

Figure I. Flowchart of the Lililwan Project timeline: Identification and characterization of the Lililwan cohort, definition of terms, culturally safe consent process utilizing "community navigators" and local languages, and results from the FASD prevalence study are detailed elsewhere. ${ }^{7-9}$ Stage I (20I0) involved interviews with I 27 (94.7\%) parents or caregivers of I34 eligible children born 2002-2003, conducted with "community navigators" trained in use of a reliable, culturally appropriate questionnaire. ${ }^{7,8}$ Stage 2 (20I0-20I I) included comprehensive multidisciplinary clinical assessments for II0 (86.6\%) of the 127 children from Stage I and development of individualized management plans. ${ }^{8,10,11}$ For Stage 3 (20I3) we mapped the available health services in the Fitzroy Valley. ${ }^{5}$ Stage 4 examined the cohorts use of the emergency department and lifetime (to age 7) hospitalizations.

(age 0-7 years) ${ }^{9}$ and existing health problems (in 2011) and ED presentations during 2007 to 2011, the focus of this paper.

\section{Emergency Department Presentations}

We retrospectively hand-searched electronic (Communicare $^{\mathrm{TM}}$ database, implemented at Fitzroy Crossing in 2007) and hard copy health records at Fitzroy Crossing Hospital for all 127 children (born 2002-3). Data extracted on ED presentations between January 1st 2007 and December 31st 2011 included the date and primary reason for presentation, outcome (managed and discharged, admitted, transferred to the nearest tertiary hospitals in Perth), and additional conditions managed at each presentation. Two doctors coded the reasons for presentation using the International Classification of Diseases Tenth Edition (ICD-10-online, World Health
Organization (WHO)), ${ }^{12}$ and entered into a Microsoft Access database. Diagnoses were classified by their main, sub, and specific code, providing 3 to 7 characters for classification. Missing data were coded as 999 . Discussion with community members and health care professionals based in Fitzroy Crossing, and review of local newspaper articles for significant regional events during this time period, informed our understanding of the local context.

\section{Data Analysis}

IBM SPSS Statistics for Mac (version 23.0.0.0 Armonk, NY, USA) was used for data analyses. Frequencies, medians, and prevalence estimates were obtained using descriptive analyses. Chi-squared tests were used to test associations between dichotomous, categorical variables, with Fisher exact test used where cell sizes were 
Table I. Emergency Department Presentations to Fitzroy Crossing Hospital, 2007-2II.

\begin{tabular}{|c|c|c|c|}
\hline \multirow[b]{2}{*}{ Emergency department presentations } & \multicolumn{3}{|c|}{ Children assessed } \\
\hline & Median & Inter-quartile range & Range \\
\hline \multicolumn{4}{|l|}{ All children $(n=127)$} \\
\hline Presentations (total $=1058$ ) & 7 & $2-13$ & $0-50$ \\
\hline ICD- 10 codes $($ total $=1743)$ & II & $3-22.5$ & $0-80$ \\
\hline \multicolumn{4}{|l|}{ Children who presented at least once $(n=103)$} \\
\hline Presentations (total $=1058$ ) & 9 & $4-14$ & $1-50$ \\
\hline ICD- 10 codes $($ total $=1743)$ & 14 & $7-25$ & $1-80$ \\
\hline \multicolumn{4}{|l|}{ Number of presentations by sex $(n=127)$} \\
\hline Males & 9 & $2-16$ & $0-50$ \\
\hline Females & 6 & $3-15$ & $0-25$ \\
\hline \multicolumn{4}{|l|}{ Presentations by prenatal alcohol exposure $(n=100)^{a}$} \\
\hline Prenatal alcohol exposure $(n=59)$ & 9 & $3.5-14$ & $1-50$ \\
\hline No prenatal alcohol exposure $(n=4 I)$ & 6 & $|-| \mid$ & $1-33$ \\
\hline \multicolumn{4}{|l|}{ Presentations for children with FASD $(n=103)^{a}$} \\
\hline Children with FASD $(n=21)$ & 9 & $3-14$ & $0-50$ \\
\hline Children without FASD $(n=82)$ & 8 & $4-14$ & $0-33$ \\
\hline Presentations by wet (October-March) and dry (May-September) seasons & Wet & Dry & Total \\
\hline 2007 & 17 & 34 & 51 \\
\hline 2008 & 106 & 64 & 170 \\
\hline 2009 & 178 & 120 & 298 \\
\hline 2010 & 125 & 140 & 265 \\
\hline 2011 & 128 & 146 & 274 \\
\hline Total & 554 & 504 & 1058 \\
\hline
\end{tabular}

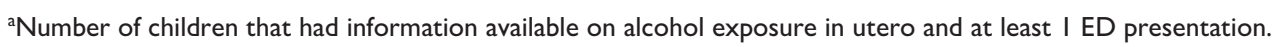

small $(<5) \cdot{ }^{13}$ Risk ratios (with $95 \%$ confidence intervals) for associations with demographic variables were calculated for children with 11-50 ED presentations and 1-10 presentations compared to children with no presentations. The 10 children who presented most often were analyzed descriptively to identify factors associated with very high presentation rates.

\section{Ethics Approval}

Stage 4, a planned extension of the Lililwan Study, was approved by the WAAboriginal Health Ethics Committee (Approval 344-04/2011); the WA Country Health Service Human Research Ethics Committee (Approval 2013:18); and the Australian National University Higher Research Ethics Committee (Approval 2016/432).

\section{Results}

The Lililwan cohort of 127 remote dwelling children born in 2002-3 comprised $94 \%$ of the primary school aged children born in 2002-3 and living in the Fitzroy Valley in 2010-11. The majority of these children were Aboriginal (95\%). The participation rate was high despite $45.7 \%$ of children living in very remote communities of less than 1000 people. Prevalence of FASD $(19 \%)$ and its co-morbidities and hospital admissions rates were high.

In 5 years $2007-11$ inclusive, there were 1058 presentations for 1743 conditions in 127 children. There were more presentations in 2009, 2010, and 2011 (274, 265, 298) than 2007 (51) and 2008 (170) with no significant difference in ED presentations numbers between the dry (May-September) and wet seasons (October-March).

Most (89.7\%) conditions were managed in the ED and children were then discharged home. Only $5.9 \%$ of presentations led to hospital admission and $4.0 \%$ were referred to external services. Less than $1.0 \%$ of children were transferred to a tertiary pediatric hospital in Perth, WA.

Of 127 children, 103 (81\%) had at least 1 ED presentation. For children who presented at least once, the median number of ED presentations, over the 5 years, was 9 (range 1-50). Of the 103 children presenting to ED, $51.2 \%$ had over 6 presentations, $32 \%$ had over 11 , and 10 had over 18 (Table 1). Based on ICD-10 codes, the median number of reasons for presentation was 14 (range $1-80, \mathrm{n}=103$ ) per child over 5 years. Males and 


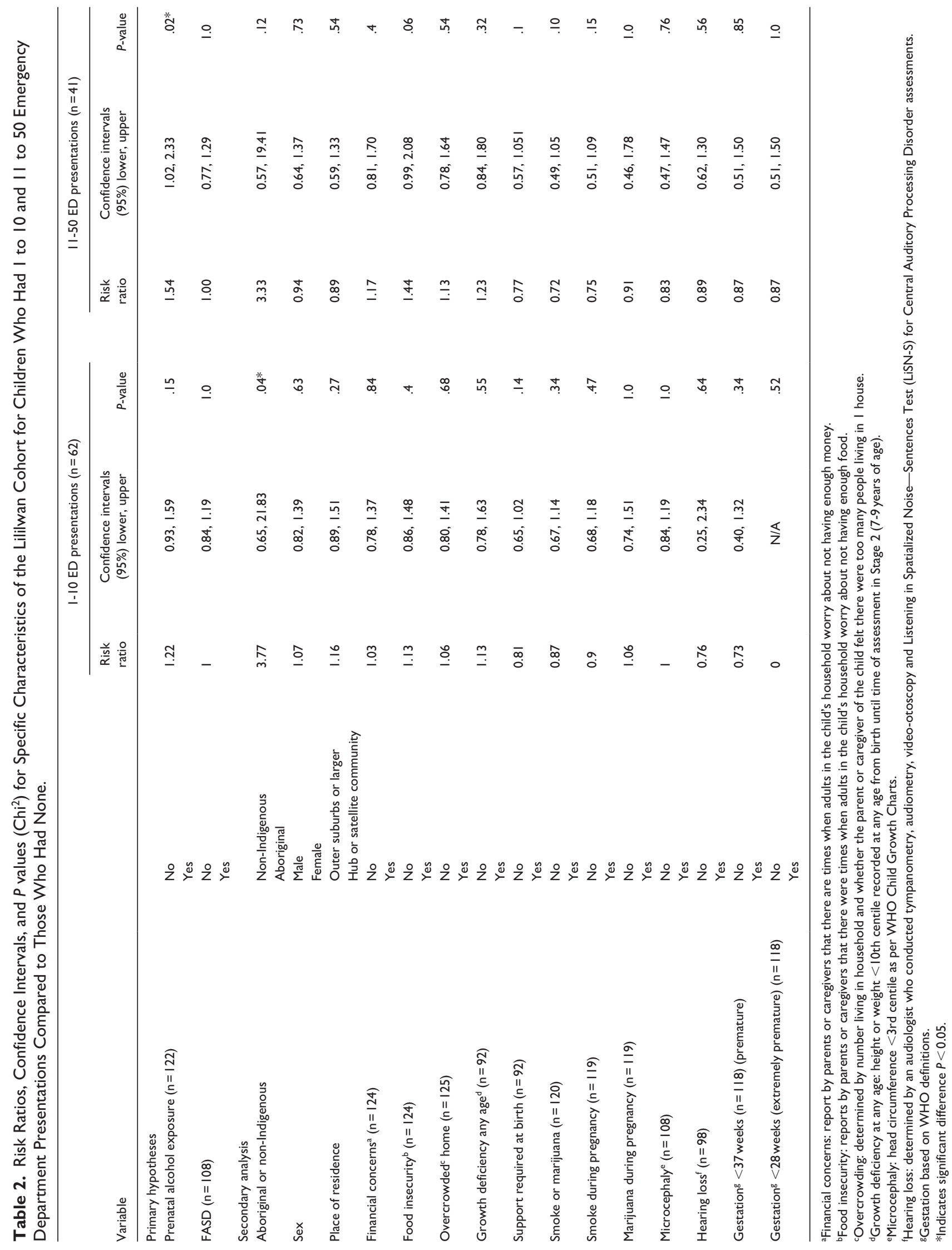


females had similar numbers of presentations (median 9 vs $6, P=0.63$ ). Children were aged 4 to 9 years at the time of ED presentation, but most $(72.2 \%)$ presentations occurred in children aged 6 years and over.

There were no significant demographic differences between children with 1 to 10 compared to 11 to 50 presentations. No difference in frequency of ED presentations was noted between children with and without FASD (Table 2).

Children with Prenatal Alcohol Exposure (PAE) $(n=59)$ had a higher number of presentations than children without PAE (median 9.0 vs 6.0, respectively), Table 1, and this was significantly associated with more frequent (11-50) than lower (1-10) presentations $(\mathrm{RR}=1.54,95 \%$ CI: 1.02-2.33, $P=0.02)$ (Table 2).

Aboriginal children ( $95.3 \%$ of 127 children) were more likely to present 1 to 10 times to the ED than nonIndigenous children ( $n=1$ vs $n=1$ vs $n=4$ for non-Indigenous children with 1-10,11-50, and no presentations, respectively) (RR 3.77, 95\% CI: 0.65-21.83, $P=0.04$ using Fisher Exact test for small sample size $(<5)$ ).

The 10 children with the highest number of presentations each had a minimum of 18 ED presentations over the 5 years. This sub-group was predominantly Aboriginal (90\%) and male (80\%) with significant existing medical issues and poor socio-economic conditions. The 10 children had 275 ED presentations (range 19-50, median 25.5) for 430 reasons (range 32-79, median 39). In these children, 167 presentations for single reasons and comorbidities existed for $39 \%$ of these presentations (range 1-4 additional comorbidities per visit). Disease of the ear $(20.4 \%)$, injury $(16.3 \%)$, skin $(15.8 \%)$, and respiratory infections $(15.4 \%)$ were the most common primary reasons for ED presentation.

The most frequent reasons for ED presentation $(n=1058)$ by ICD10 chapter code were conditions coded as "factors influencing health status and contact with health services" (16.0\%) (Table 3$)$. These are typically deemed primary care conditions, usually seen in a GP clinic. For example: continued care for an ongoing ailment, vaccination, issues influencing health status, or problems not classified as disease or injury, for example, social issues. In our study, these presentations included sub-acute follow up of a condition ( $47.3 \%$ of these ICD chapter presentations, $7.6 \%$ of all presentations), hearing or ear checks $(23.1 \%, 3.7 \%)$, screening (eg, for STIs, trachoma, anemia, diabetes) $(13 \%, 2.0 \%)$, and growth checks $(10.1 \%, 1.6 \%)$.

Injury or poisoning represented $15.1 \%$ of presentations. Of the injuries, wounds accounted for $48.0 \%$ of presentations (and $7.6 \%$ of all presentations), fractures and dislocations for $12.1 \%(1.9 \%)$, head injury with concussion $9.0 \%$ (1.42\%) (Table 3).
In the group with between 11 and 50 presentations, wounds were responsible for $8.6 \%$ of injury presentations compared to $5.3 \%$ for the group with 1 to 10 presentations. All other injuries were proportionally more frequent in the lower presentation group (1-10) than the higher presentation rate group (11-50).

Diseases of the skin and subcutaneous tissue comprised $13.8 \%$ of all ED presentations and the majority 91.1\% were infections (which alone accounted for $12.6 \%$ of all presentations). Diseases of the ear and mastoid process, mostly otitis media, accounted for $14.9 \%$ of presentations. Furthermore, $13.4 \%$ of ED presentations were for diseases of the respiratory system including upper and lower respiratory tract infections (Table 3).

Of all ED presentations $(n=1058), 50.9 \%$ were the direct result of infection (47.7\% in the group with 11-50 admissions and $57.7 \%$ in the group with $1-10$ admissions). Almost two thirds (63.8\%) of infectious presentations occurred within the high presentation group (11-50).

Of ED presentations, 9.8\% were for infectious and parasitic diseases, for example, gastroenteritis $(39.4 \%$ of infectious or parasitic disease, $\mathrm{n}=41)$, trachoma $(28.9 \%$, $\mathrm{n}=30$ ), strongyloidiasis $(\mathrm{n}=6)$, meningococcal meningitis $(n=2)$, fungal infections such as tinea $(n=5)$, head lice $(n=3)$, scabies $(n=3)$, cutaneous larva migrans (hookworm) $(\mathrm{n}=3)$, and necrotizing ulcerative stomatitis $(n=1)$ (Table 3$)$. Other symptoms, signs and abnormal laboratory or clinical findings (eg, cough, fecal incontinence, headache, lethargy, edema, failure to thrive) accounted for $6.8 \%$ of presentations.

Presentation rates did not differ significantly for any ICD chapter code between groups with low (1-10) and high (11-50) numbers of presentations.

\section{Discussion}

Over a 5-year period, there were high numbers of ED presentations in a cohort of remote dwelling, predominantly Aboriginal children. Most (82\%) children presented at least once and a third (32\%) had more than 11 presentations over 5 years. Presentations $(n=1058)$ were typically for: infections of skin ( $12.5 \%$ of total presentations), middle ear (12.2\%) and upper respiratory tract (9.8\%); follow-up (7.5\%); and wounds (7.5\%). The high rate $(89.7 \%)$ of discharges home after management in ED suggests low acuity presentations. Many of these children could potentially have been seen by GPs or community health workers, however such services were limited, necessitating ED use. There was an increase in the number of presentations during 2009-11 compared to 2007-8, but seasonality did not affect numbers. There 


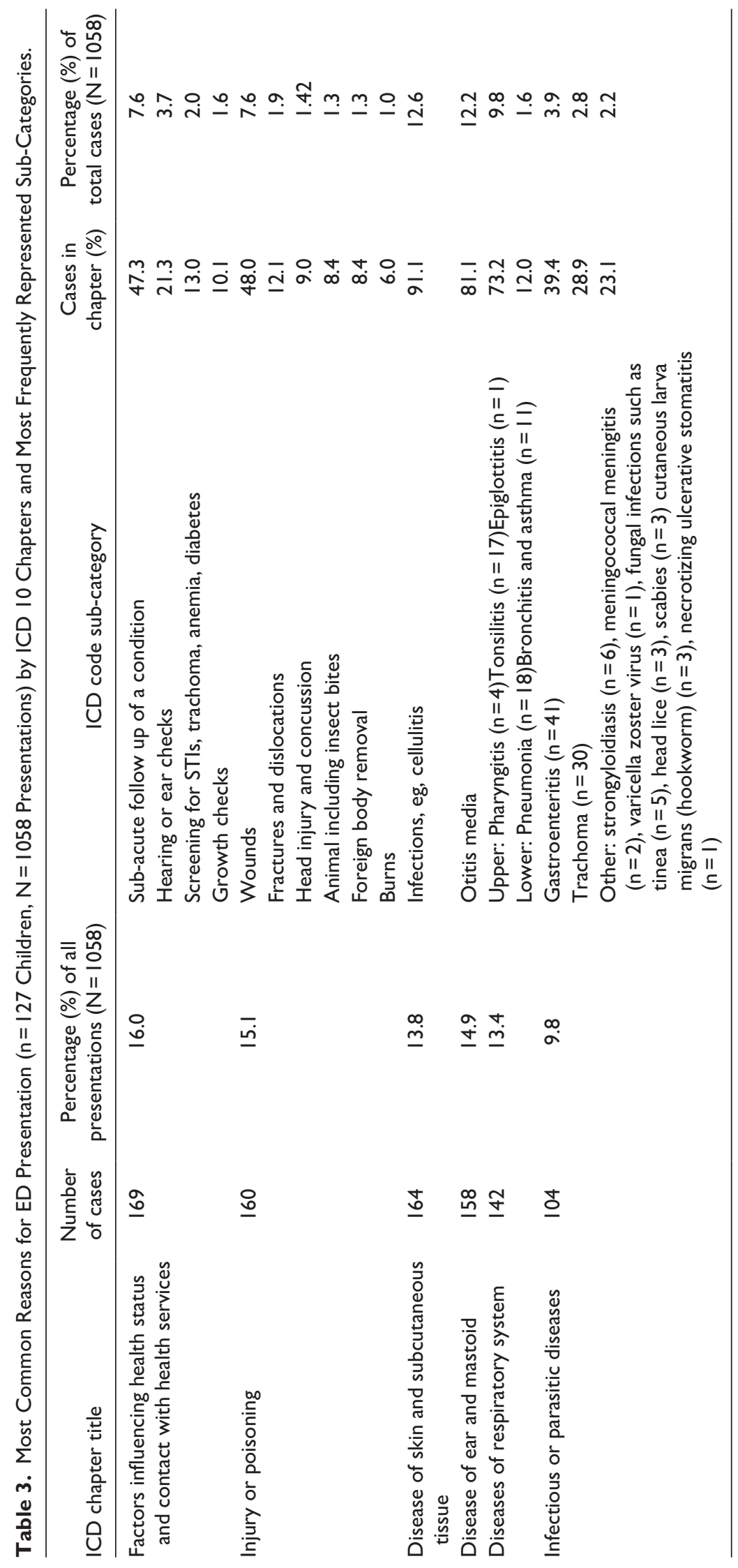


were higher numbers of presentations in children with PAE and in Aboriginal children but no association with age or gender.

Reflecting the population in the Fitzroy Valley, a higher proportion of children in our study $(95.3 \%)$ was Aboriginal compared to the proportion in studies from tertiary hospitals in Australia and New Zealand (3\%) $)^{14}$ and the Northern Territory (31\%). ${ }^{15}$ However, the reasons for presentation were similar, most commonly acute, viral gastrointestinal, and respiratory infections. Similar to the NT study, ${ }^{15}$ injury and skin infections were common reasons for presentation in the Fitzroy Valley but in our young children acute psychiatric presentations were not well-documented. Our findings are also consistent with previous studies that examined reasons for Aboriginal child presentations to remote primary health care services in the Northern Territory (NT) ${ }^{16}$ and Sydney. ${ }^{17}$

Over half $(50.9 \%)$ of presentations in our cohort were for potentially preventable infectious conditions, consistent with common causes for ED presentation for Aboriginal people of all ages in the Kimberley in 20089. ${ }^{18}$ The 2004 Aboriginal Child Health Survey of children aged 0 to 17 living in WA, provides congruent data and shows that infectious conditions were highest in young children. ${ }^{19}$

Infectious diseases are closely interlinked with poverty. Poverty contributes to a spiral of lifelong adverse outcomes including social stigma, poor housing and sanitation, low education levels, unemployment, and poor nutrition which in turn increase the risk of acute and chronic illness and disability. ${ }^{20}$ As discussed in the Kimberley Aboriginal Primary Health Plan 2012-15, many infections could be prevented by better living conditions, including reducing overcrowding and ensuring clean drinking water, sanitation, and vaccination. ${ }^{18}$ Many presenting conditions in our cohort (eg, hookworm, strongyloidiasis, meningococcal meningitis, trachoma, and rotavirus gastroenteritis) have been almost eradicated elsewhere in Australia.

Hygiene and education programs should be developed and managed by primary health care and education systems. ${ }^{18}$ In Fitzroy Crossing, Nindilingarri Cultural Health Services play an important role in health promotion. Skin and throat infections are common in this population and sometimes lead to systemic infections and rheumatic heart disease. ${ }^{21}$ Since we commenced our study, the Telethon Kids Institute with Western Australia Country Health Service (WACHS) and Kimberley Aboriginal Medical Services (KAMS), initiated the SToP Program (see, Treat and Prevent skin sores and scabies) in the Kimberley region with the intention of decreasing skin infection rates to under $5 \% .{ }^{22}$ There is also a "no school, no pool" rule; the chlorinated pool decreases rates of otitis media and scabies. ${ }^{23}$

Strong coverage from immunization programs prevent infectious diseases and their spread. Children in the Lililwan cohort were eligible for free vaccination under the National Immunisation Program ${ }^{24}$ however we did not obtain immunization records for this cohort. In 2019, the national coverage rate for vaccinations in 5-year-old Indigenous Australian children was 96.8\%. ${ }^{25}$

Injury rates could be decreased using culturally appropriate preventative education for leading causes and complications of injury in Fitzroy Crossing (eg, head injuries, burns, and snake bite), and awareness of non-accidental injuries. ${ }^{26}$ Interventions should include health and safety promotion including hygiene, wound care, and improved environmental safety. ${ }^{26}$

The potential reasons for increased ED presentations from 2009 to 2011 include decreased access to primary health care in the community ${ }^{5}$ and increased health literacy. Health literacy is an individual's capacity to understand and use health information to meet health needs. ${ }^{27}$ Poor health literacy in parents is associated with increased and inappropriate engagement with EDs. ${ }^{27}$ Community-led alcohol restrictions and education programs were implemented in September 2007; the Marulu strategy commenced in 2008; and Stages $1 \& 2$ of the Lililwan Project were completed in 200912. These activities included culturally appropriate health education in local Aboriginal languages, which likely improved parents' awareness about child health and wellbeing and their willingness to engage with health services, compared to 2007-8. Children with PAE (49\%) had higher rates of ED presentations compared to children with no PAE and this may reflect issues associated with continued alcohol use at home.

When primary health care services are limited and access is difficult, for example, in this very remote context with extreme climate and limited transport, use of the emergency department becomes a proxy for primary health care use, particularly for conditions that would be deemed low acuity at triage and should be treated in the community. ${ }^{18}$ Our data is supported by WACHS 2018 report which demonstrates that from 2011 to 2015 the majority (74\%) of Kimberley wide ED attendances were triage $4 / 5$ (which is indicative of issues that could be managed by primary health care services) and that similar challenges occur in other remote communities across the Kimberley. ${ }^{4}$ Despite discussions with local health care workers and review of reports from this time period, it was difficult to accurately ascertain the numbers of GPs or remote community nurse practitioners servicing the Fitzroy Valley over the 5-year period 2007-11. ${ }^{9}$ Our 2013 review of child health services 
showed high rates of potentially preventable hospital admissions. ${ }^{9}$ A previous NSW study demonstrated that absence of a GP increased ED presentations for low acuity patient presentations by up to $54.7 \%$ in inner regional areas and that the number of presentations was higher in younger patients. ${ }^{28}$ At some periods during our study, due to a scarcity of doctors, the ED was staffed by nurses only who did not document diagnoses. Since our data were collected, the Fitzroy Crossing ED has undergone restructuring in an attempt to encourage low acuity patient presentations out of the ED into GP clinics. This followed community consultation and increased GP attendance rates from 67\% in August 2010-11 to 85\% in August 2011-March 2012, accompanied by a decrease by about $300 \%$ in lower acuity triage presentations to ED. ${ }^{18}$

Environmental factors, including a large dust storm, cyclone Yvette, and the fire that burnt down the Fitzroy Crossing Shopping Centre in July 2009 may explain reduced ED presentations that year. The similar number of ED presentations in the dry and wet seasons was surprising, considering the wet season deems roads impassable for weeks. However, there are year-round barriers to accessing health service including long distances, few private cars or drivers and a lack of public transport that may account for this stability across seasons. ${ }^{5}$

Although our study is limited by a lack of data on triage categories and the challenge of not having an age matched comparative cohort, we believe it is important as it documents use of a hospital ED over 5 years by a near complete, population-based sample of predominantly Aboriginal children in a very remote setting. This study is strengthened by the lack of selection bias and the fact that ED use in Fitzroy Crossing is likely representative of similar remote-dwelling cohorts in Australia. The high rates of ED use indicate need for better preventative strategies for infection and injury, identifies gaps in primary care services, and could inform policy and practice in this and other remote communities.

This research was requested and led by Aboriginal women leaders in the community following years of community consultation. We achieved high participation rates despite vast geographical distances, due to use of community navigators (respected locals who brokered all contacts between researchers and communities). A validated research tool was used to collect information on PAE and a reliable and culturally appropriate questionnaire was used to obtain demographic details from families. ${ }^{8,11}$

The Kimberley population is the fastest growing population in WA, expanding at an average rate of $2.7 \%$ per annum. ${ }^{29}$ The skewed Aboriginal Australian population pyramid, in favor of young people (56\% aged 0-24 years (2006 Australian Census)), means an ongoing and increasing demand for child health services in the Kimberley Region. ${ }^{18}$ This paper adds novel data on the context and underlying reasons for ED presentations for children in Fitzroy Crossing and will inform educational tools and development of adequate infrastructure to better support these communities and similar remote communities in Australia and overseas. We have previously demonstrated high rates of disability including FASD, ${ }^{6}$ high rates of hospital admissions, ${ }^{9}$ and limited child health services in the region. ${ }^{5}$ We have also documented modifiable determinants of ill-health, including poor housing and sanitation and the need for clean water, parental education, and health literacy. Knowledge of health needs and service gaps, both preventive and clinical, will allow future service planning, in consultation with and ideally driven by the local population, to improve child health and well-being.

\section{Acknowledgments}

The authors acknowledge members of the Lililwan Project team who contributed to this work: Dr James Fitzpatrick, Professor John Boulton, Professor Carol Bower, Sharon Eadie, Robyn Doney, Barbara Lucas, Jane Latimer, Olive Knight, Juliette O'Brien, Dr Melanie Olding, and Charlie Schmidt. Particular thanks go to members of the Fitzroy Valley communities, Marulu FASD Strategy leadership team and staff of Nindilingarri Cultural Health Services and Marninwarntikura Women's Resource Centre. We would like to acknowledge Rochelle Watkins for her insight and assistance with statistical analyses.

\section{Author Contributions}

PJD performed the research, analyzed the data, interpreted the results, drafted the manuscript, read and approved final manuscript. EFMF performed the research, collecting and interpreting the data, proofed and approved the final manuscript. KG interpreted results, drafted manuscript, and read and approved final manuscript. RW analyzed the data. JO conceived the study, designed the study, and performed the research. MC conceived the study, designed the study, and performed the research. HEJ interpreted the results, drafted the manuscript, and read and approved the final manuscript. KD interpreted the results, drafted the manuscript, and read and approved the final manuscript. DH interpreted the results, drafted the manuscript, and read and approved the final manuscript. EJE conceived the study, designed the study, performed the research, read and approved the final manuscript. ALCM conceived the study, designed the study, and performed the research, read, reviewed, edited and approved the final manuscript. All authors have contributed to and approved the final manuscript. 


\section{Declaration of Conflicting Interests}

The author(s) declared no potential conflicts of interest with respect to the research, authorship, and/or publication of this article.

\section{Funding}

The author(s) disclosed receipt of the following financial support for the research, authorship, and/or publication of this article: The Lililwan project is supported by the National Health and Medical Research Council of Australia (NHMRC) (Elizabeth Elliott, Practitioner Fellowships 457084 and 1021480, and project grant 1024474); the Australian Research Council (Jane Latimer, Future Fellowship 0130007); the Australian Government Departments of Health and Ageing (DoHA); and Families, Housing, Community Services and Indigenous Affairs (FaHCSIA); Save the Children Australia, the Foundation for Alcohol Research and Education and the University of Sydney Poche Institute (Philippa Dossetor, Poche Scholarship). Pro bono support has been provided by M\&C Saatchi, Blake Dawson solicitors, and the Australian Human Rights Commission. Alexandra Martiniuk was funded by an NHMRC TRIP (Translating Research into Practice) Fellowship (2016-2018). Philippa Dossetor is supported by a part-time $\mathrm{PhD}$ scholarship through the Australian National University College of Medicine, Biology and Environment and by a part-time Avant Doctors-in-Training scholarship.

\section{ORCID iD}

Philippa Jane Dossetor (iD https://orcid.org/0000-00022226-0693

\section{References}

1. Australian Institute for Health and Welfare. The Health and Welfare of Australia's Aboriginal and Torres Strait Islander Peoples 2015. Australian Government; 2015.

2. Prime Minister's Report. Closing the Gap: The Prime Minister's Report. National Indigenous Australians Agency; 2017.

3. Morphy F. Population, People and Place: The Fitzroy Valley Population Project. Australian National University, Centre for Aboriginal Economic Policy Research; 2010.

4. Services WCH. Kimberley Health Profile. WA Country Health Service; 2018.

5. Dossetor PJ, Thorburn K, Oscar J, et al. Review of Aboriginal child health services in remote Western Australia identifies challenges and informs solutions. BMC Health Serv Res. 2019;19:758. doi:10.1186/s12913019-4605-0.

6. Fitzpatrick JP, Latimer J, Carter M, et al. Prevalence of fetal alcohol syndrome in a population-based sample of children living in remote Australia: the Lililwan* Project. J Paediatr Child Health. 2014;51:450-457. doi:10.1111/ jpc.12913_12.

7. Latimer J, Elliott E and Carter M. Marulu: The Lililwan Project. Report no. 978-0-646-53390-2. The George Institute for Global Health; 2010.
8. Fitzpatrick JP, Elliott EJ, Latimer J, et al. The Lililwan Project: study protocol for a population-based active case ascertainment study of the prevalence of fetal alcohol spectrum disorders (FASD) in remote Australian Aboriginal communities. BMJ Open. 2012;2:e000968. doi:10.1136/bmjopen-2012-000968.

9. Dossetor PJ, Martiniuk ALC, Fitzpatrick JP, et al. Pediatric hospital admissions in Indigenous children: a population-based study in remote Australia. BMC Pediatr. 2017;17:195. doi: 10.1186/s12887-017-0947-0.

10. Elliott E, Latimer J, Oscar J, et al. The Lililwan Collaboration: Inquiry into Fetal Alcohol Spectrum Disorders (FASD). Report no. 978-0-9873014-0-6 and 978-0-9873014-1-3; 2012.

11. Fitzpatrick JP LJ, Ferreira ML, Carter M, Oscar J, Martiniuk AL, Watkins RE, Elliott EJ. Prevalence and patterns of alcohol use in pregnancy in remote Western Australian communities: The Lililwan Project. Drug Alcohol Rev. 2015;34:329-339. doi:10.1111/dar.12232.

12. National Centre for Classification in Health. The International Statistical Classification of Diseases and Related Health Problems, 10th revision, Australian Modification (ICD-10-AM). National Centre for Classification in Health; 2000.

13. Why does Fisher's exact test disagree with the confidence interval for the odds ratio? 1996-2019. Updated 2019. Accessed 30 July, 2020. https://www.stata.com/support/ faqs/statistics/fishers-exact-test/

14. Acworth J, Babl F, Borland M, et al. Patterns of presentation to the Australian and New Zealand paediatric emergency research network. Emerg Med Australas. 2009;21:59-66. doi:10.1111/j.1742-6723.2009.01154.x.

15. Buntsma D LA, O'Neill E, Palmer D, Morris P, Acworth J, Babl FE; Paediatric Research in Emergency Departments International Collaborative (PREDICT). Patterns of paediatric emergency presentations to a tertiary referral centre in the Northern Territory. Emerg Med Australas. 2017;29:678-685. doi:10.1111/1742-6723.12853.

16. Bar-Zeev SJ, Kruske SG, Barclay LM, et al. Use of health services by remote dwelling Aboriginal infants in tropical northern Australia: a retrospecitve cohort study. BMC Pediatr. 2012;12:19. DOI: https://doi.org/10.1186/14712431-12-19.

17. Duncan C, Williams K, Nathanson D, et al. Emergency department presentations by Aboriginal children: issues for consideration for appropriate health services. J Paediatr Child Health 2013;49:E448-E450. doi:10.1111/jpc.12225.

18. Lewis J. Kimberley Aboriginal Primary Health Plan 2012-2015. Kimberley Aboriginal Health Planning Forum; 2013.

19. Zubrick SR LD, Silburn SR, Blair E, Milroy H, Wilkes T, Eades S, D'Antoine H, Read A, Ishiguchi P, Doyle S. The Western Australian Aboriginal Child Health Survey: The Health of Aboriginal Children and Young People. Telethon Institute for Child Health Research; 2004.

20. Bhutta ZA, Sommerfeld J, Lassi ZS, Salam RA, Das JK. Global burden, distribution, and interventions for infectious diseases of poverty. Infect Dis Poverty 2014;3:21. 
21. Murdoch J, Davis S, Forrester J, et al. Acute rheumatic fever and rheumatic heart disease in the Kimberley: using hospitalisation data to find cases and describe trends. Aust N Z J Public Health. 2014;39:38-43. doi:10.1111/17536405.12240

22. Telethon Kids Institute. Program aims to stop skin infections in their tracks. July 4, 2017.

23. Lehmann D, Tennant MT, Silva DT, et al. Benefits of swimming pools in two remote Aboriginal communities in Western Australia: intervention study. BMJ. 2003:327: 415-419. doi:10.1136/bmj.327.7412.415

24. National Centre for Immunisation Research and Surveillance. Vaccination History Tables. The University of Sydney; 2002-2015.

25. Commonwealth of Australia. Health topics. 2019. Updated 2019. Accessed 28 July, 2020. https://www.health.gov. $\mathrm{au} /$ health-topics/immunisation/childhood-immunisationcoverage/immunisation-coverage-rates-for-aboriginaland-torres-strait-islander-children

26. National Public Health Partnership. The National Injury Prevention and Safety Promotion Plan: 2004-14. Commonwealth of Australia; 2004.

27. Morrison AK, Myrvik MP, Brousseau DC, et al. The relationship between parent health literacy and pediatric emergency department utilization: a systematic review. Acad Pediatr. 2013;13:421-429. doi:10.1016/j.acap.2013.03.001

28. Stephens AS, Broome RA. Patterns of low acuity patient presentations to emergency departments in New South Wales, Australia.Emerg Med Australas. 2017;29:283-290. doi:10.1111/1742-6723.12767.

29. Department of Health. Aboriginal Health Profile: Kimberley Health Region. Department of Health; 2009. 